\title{
Le PROFESSEUR LOUIS ROULE
}

\author{
Par le Docteur Jacques PELlEgRIN, \\ Professeur au Museum National d'Histoire Naturelle
}

Le Docteur Louis Roule, professeur honoraire au Muséum national d'histoire.naturelle, est mort presque subitemeñ à Versailles, le 30 Juillet 1942,

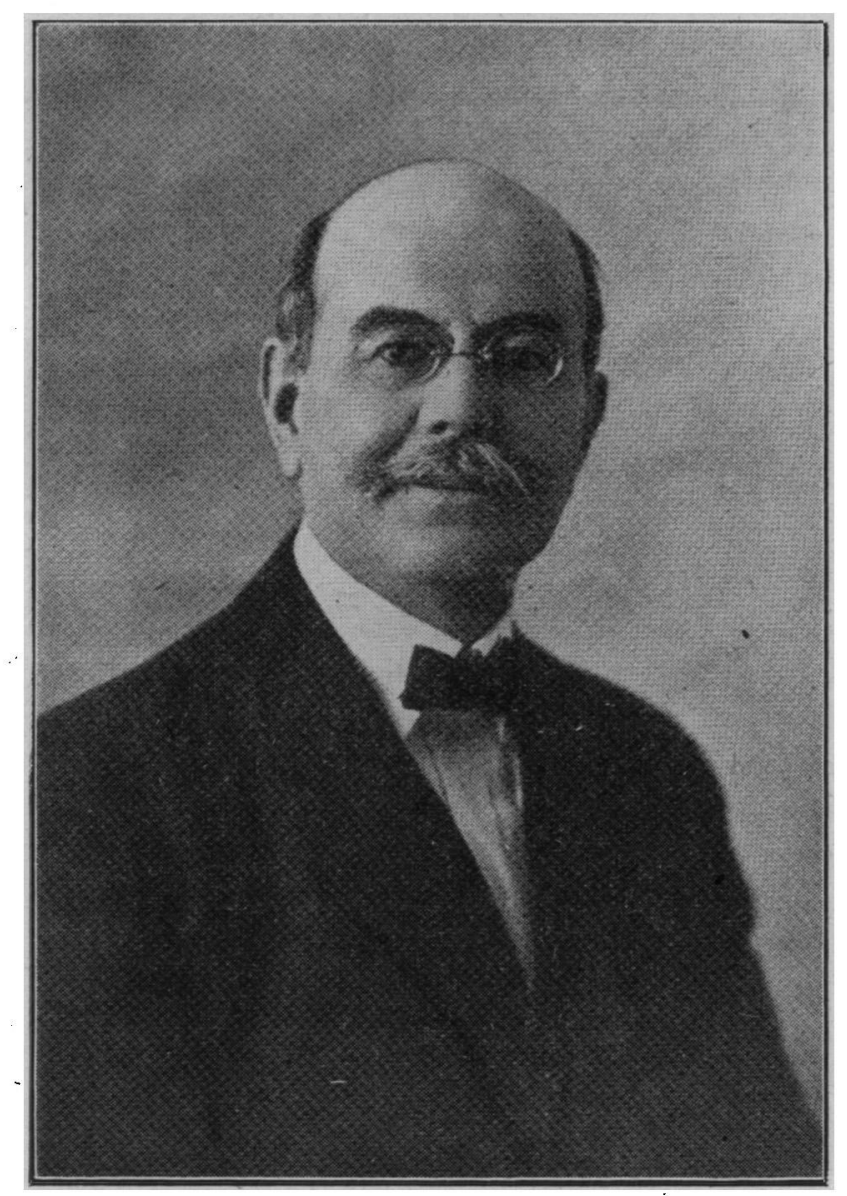

Frc. 3. - Le Professeur Louis Roule, 1861-1942.

dans sa $81^{\circ}$ année. L'inhumation a eu lieu au cimetière du Père-Lachaise, dans le caveau de famille, le 3 Aout, à $15 \mathrm{~h}$. 30, en présence d'une nombreuse assistance parmi laquelle on remarquait la plupart des professeurs 
du Muséum, avec, à leur tête, le Professeur Urbain, assesseur. C'est à moi que revint l'honneur de prononcer le discours d'usage sur la tombe de mon distingué prédécesseur. Seule $M^{\text {mo }}$ Roure et sa plus jeune fille Lucie avaient pu se rendre aux obsèques du regretté défunt. Le reste de la famille, en effet, est dispersé de par le monde; son gendre et sa fille afnée, $M$. et $\mathbf{M}^{\text {mo }}$. Masson, sont au Brésil ; son fils Frédéric est fonctionnaire en Indochine, qu'il habite avec les siens, et sa fille Sużanne exerce la médecine au Maroc.

Il me faut maintenant retracer les étapes de la longue et féconde carrière du savant qui disparait en insistant sur ses principaux travaux et particulièrement ici sur ceux qui concernent' l'ichthyologie et la pisciculture.

Louis Roule était né à Marseille, le 20 Décembre 1861.

Attiré de bonne heure par l'étude des sciences biologiques et par l'enseignement, il fut nommé, dès 1881 , chef de travaux pratiques à l'Ecole de Médecine de Marseille, passa sa thèse de doctorat ès sciences naturelles en 1884, sur les Ascidies des côtes de Provence ; en 1891, celle de docteur en médecine avec un mémoire sur la structure du tissu musculaire. En 1885, il fut nommé maftre de conférences à la Faculté des Sciences de Toulouse, il y passa professeur en 1892 à 30 ans à peine; en 1903 , il devint conservateur du Musée d'histoire naturelle du chef-lieu de la Haute-Garonne. C'est en 1910 qu'il arriva à Paris comme professeur de la chaire de Zoologie (Reptiles, Batraciens et Poissons) du Muséum national d'histoire naturelle. Il devait l'occuper jusqu'en 1937, c'est-à-dire pendant plus d'un quart de siècle. En 1925, il fut chargé d'une maîtrise de conférences de pisciculture à l'Institut national agronomique et dirigea le laboratoire d'ichthyologie générale et appliquée de l'Ecole des Hautes Etudes, rattaché à sa chaire au Muséum.

L'œuvre de Louis Roule est considérable. Ses premiers travaux portent surtout sur les Invertébrés : Vers et Tuniciers. Il publie d'importants traités concernant l'embryologie et l'anatomie comparée. Ceṕendant, la direction de la Station de pisciculture et d'hydrobiologie de Toulouse, qui lui est confiée en 1901, le met déjà en contact avec nos principales espèces de Poissons indigènes ou acclimatées.

A partir de sa nomination, en 1910, au Muséum, il se consacre désormais plus spécialement à l'ichthyologie, étudiant surtout les Poissons des grandes profondeurs, revoyant nombre de collections récoltées par le Prince Albert I ${ }^{\text {r }}$ de Monaco, le Docteur Charcot dans l'Antarctique, le savant danois Johannes Schmid, concernant les Anguilles. Pour n'en citer qu'un exemple, c'est à Roule qu'on doit la première description du Grimaldichthys profundissimus, qui bat le record des Poissons abyssaux, ayant été remonté d'une profondeur de plus de 6 kilomètres, soit exactement de 6.035 mètres.

Son gout pour l'embryologie le poùsse aussi $\grave{a}$ des recherches concer- 
nant les formes larvaires et le développement de Poissons de différents groupes.

Un autre de ses sujets de prédilection est l'étude des espèces migratrices, aussi bien celles qui passent une partie de leur existence dans les eaux douces, l'autre dans les eaux salées comme le Saumon atlantique, l'Esturgeon, les Aloses, que celles exclusivement marines comme le Thon de la Méditerranée.

Mais toute une partie de son enseignement et de ses travaux est orientée vers des buts pratiques, et il y a lieu spécialement de le faire remarquer ici, l'aquiculture a toujours été une de sos préoccupations constantes. C'est ainsi qu'en 1914 il publie un volumineux " Traité raisonné de la pisciculture et des pêches " qui renferme une foule de renseignements précieux. Il lui consacre encore par la suite de nombreux mémoires ou petits ouvrages, comme son intéressant volume sur " les Poissons des eaux douces de la France " (1925), comme son utile "Manuel de pisciculture " (1932), publié dans l'Encyclopédie des connaissances agricoles et où les plus récentes méthodes de carpiculture et de trutticulture sont exposées avec clarté et précision. Enfin, il entreprend et mène à bien la publication d'un vaste ouvrage, sorte d'encyclopédie ichtyologique en 10 volumes, artistiquement illustrés par Fernand Angel et ayant pour titre : " Les Poissons et le monde vivant des eaux ". L'ayant commencé en 1926, il l'achève en 1937, mettant ainsi l'étude des Poissons à la portée de tous, vulgarisant les méthodes les plus pratiques de l'exploitation des eaux marines et fluviales.

Louis Roule fut également historien et critique. C'est ainsi qu'on lui doit une série de petits volumes consacrés à la vie et à l'œuvre des grands naturalistes qui illustrèrent le jardin du Roi et le Muséum : Buffon, Bernardin de Saint-Pierne, Daubenton, Lamarck, Lacépède, Cuvier.

Une si longue carrière et si bien. remplie, poursuivie avec tant de continuité et de persévérance, devait avoir ses récompenses.

Louis Roule était commandeur de la Légion d'honneur, membre de l'Académie d'Agriculture, plusieurs fois lauréat de l'Académie des Sciences (grand prix des Sciences physiques 1889 et 1919, prix Serres 1899). Il présida de nọmbreuses Sociétés soientifiques, par exemple la Société Zoologique (1913), la Société d'Aquiculture (1920), il prit part à divers congrès tant en France qu'à l'étranger, fut chargé à plusieurs reprises de missions, notamment en ce qui concerne l'inspection des Musées provinciaux d'histoire naturelle. C'est ainsi qu'il joua un rôle important dans la fondation de l'Association des Conservateurs de Collections publiques de France.

Puisse cette trop rapide esquisse donner une idée d'ensemble de la variété et de l'étendue des travaux du maltre regretté qui vient de disparaître. 